\title{
The Buckling of Fuel Rods under Inertia Loading
}

\author{
Gordon S. Bjorkman \\ Spent Fuel Storage and Transportation Division, U.S. Nuclear Regulatory Commission
}

\begin{abstract}
The buckling analysis of fuel rods during an end drop impact of a spent fuel transportation cask has traditionally been performed to demonstrate the structural integrity of the fuel rod cladding or the integrity of the fuel geometry in criticality evaluations for a cask drop event. The actual calculation of the fuel rod buckling load, however, has been the subject of some controversy, with estimates of the critical buckling load differing by as much as a factor of 5. Typically, in the buckling analysis of a fuel rod, assumptions are made regarding the percentage of fuel mass that is bonded to or that participates with the cladding during the buckling process, with estimates ranging from 0 to $100 \%$. The greater the percentage of fuel mass that is assumed to be bonded to the cladding the higher the inertia loads on the cladding, and, therefore, the lower the "g" value at which buckling occurs. However, these solutions do not consider displacement compatibility between the fuel and the cladding during the buckling process. By invoking displacement compatibility between the fuel column and the cladding column, this paper presents an exact solution for the buckling of fuel rods under inertia loading. The results show that the critical inertia load magnitude for the buckling of a fuel rod depends on the weight of the cladding and the total weight of the fuel, regardless of the percentage of fuel mass that is assumed to be attached to or participate with the cladding in the buckling process. Therefore, $100 \%$ of the fuel always participates in the buckling of a fuel rod under inertia loading.
\end{abstract}

\section{INTRODUCTION}

Casks designed to transport new and spent nuclear fuel are required to meet strict government requirements to demonstrate their structural integrity and the capability to remain leaktight after a severe accident event. One of the hypothetical accident conditions to which the cask is subjected, as prescribed in 10 CFR Part 71 [1], is a free drop through a distance of 9 meters (30 feet) onto an unyielding surface in a position in which maximum damage is expected. To ensure compliance, multiple drops in different orientations are performed.

Fuel rod buckling analyses under bottom end drop impact and center of gravity (c.g.) over corner impact have traditionally been performed to demonstrate the structural integrity of the cladding and the integrity of the fuel geometry (pitch) in criticality evaluations. Buckling analyses are performed for both fresh fuel and spent fuel. A typical PWR fresh fuel rod might be composed of a cladding tube 4 meters long with an outside diameter and wall thickness of $1.00 \mathrm{~cm}$ and $0.06 \mathrm{~cm}$ respectively. The cladding tube is filled with fuel pellets about $2 \mathrm{~cm}$ long with a diameter slightly less than the inside diameter of the cladding. The fresh fuel pellets are stacked one on top of the other and are not bonded to the cladding. The stack of fresh fuel pellets then acts as a column made from a material with high compressive strength but no tensile strength. In the case of spent fuel the irradiation process changes the mechanical properties of both the cladding and fuel, and causes some of the fuel to bond to the cladding.

Lawrence Livermore National Laboratory (LLNL) and Sandia National Laboratory (SNL) have published analytical methods to calculate the buckling load of a fuel rod for an end drop impact. Both methods use only the bending stiffness of the cladding which is reasonable given the uncertainties in determining the bending stiffness of spent fuel. The analytical method described by LLNL [2] assumes that buckling occurs when a fuel rod segment between the bottom two spacer grids reaches the Euler buckling limit. The analytical method uses material properties for irradiated cladding, considers the weight of the cladding, but neglects the weight of fuel pellets.

In the buckling analysis method used by SNL [3], assumptions are made regarding the amount of fuel mass that is bonded to and participates with the cladding during the buckling process. The greater the amount of fuel mass participating with the cladding the higher the inertia loads on the cladding, and therefore, the lower the "g" value at which buckling occurs. The SNL report states:

"For the buckling analysis, only the percentage of fuel pellet mass attached to the cladding is included in the fuel response model. Mass not connected to the cladding is assumed to be supported independently of the fuel rod cladding: 75\% of the PWR fuel mass and 10\% of the BWR fuel mass are used in the analysis." 
In the case of the BWR fuel rod, $10 \%$ of the mass of the fuel is added to the mass of the cladding, and the number of "g's" required to cause buckling of the cladding is calculated. The $90 \%$ of the fuel mass not attached to the cladding, and supported independently of the cladding, is neglected.

The NRC staff does not agree with either of these approaches with regard to the treatment of fuel mass. The NRC [4,5] considers that in addition to the weight of the cladding, buckling analyses of fuel rods under inertia loading should include the total weight of fuel pellets. This guidance was thought to be very conservative, but as will be shown herein, was completely appropriate.

\section{CRUX OF THE PROBLEM}

A fuel rod can be thought of simply as a column of fuel inside a cladding tube (column). The problem with the LLNL and SNL methodologies is that they do not incorporate a fundamental behavior of this simple model, i.e., displacement compatibility between the fuel column and the cladding column. As will be shown herein, this "independently supported fuel" interacts with the cladding and in so doing significantly reduces the critical buckling load of the fuel rod. The fuel mass that is not attached to the cladding, which has been neglected in both approaches, fully participates in the buckling process.

The solution for the buckling of a fuel rod under inertia loading will be approached first qualitatively and then quantitatively.

\section{QUALITATIVE EVALUATION OF FUEL ROD BUCKLING}

Consider the buckling of a fuel pellet column inside a cladding tube column, i.e., dowel inside a circular tube, where the fuel is not bonded to the cladding. Since the fuel column contains approximately $80 \%$ of the mass (inertia load) of the fuel rod, assume, for the sake of simplicity, that the cladding column is weightless relative to the fuel column. Thus, the fuel column contains the entire inertia load in the problem, and there is no inertia load acting on the cladding column.

For the idealized problem described above, how does buckling take place in the three cases of displacement compatibility considered below?

Case 1: Lateral displacement compatibility between the fuel column and cladding column is NOT required.

In this case, let the inertia load (g load) increase until the fuel column reaches its buckling load. Since displacement compatibility between the fuel column and cladding column is not maintained, the fuel column is allowed to buckle and displace laterally beyond the boundary of the cladding column. The cladding column, on the other hand, does not "feel" the lateral movement of the fuel column and remains in place in its original position. Because the cladding column is weightless, its buckling load is infinite (or very high, if it were not assumed to be weightless). This idealized case dramatizes the buckling behavior that occurs in the LLNL and SNL methodologies.

Case 2: Lateral displacement compatibility between the fuel column and cladding column is required.

The g loading is increased until the fuel column reaches its previous (Case 1) buckling load. But now the fuel column cannot buckle at this load because the cladding column is restraining it from moving laterally. The bending stiffness of the combined system is the bending stiffness of the fuel column plus the bending stiffness of the cladding column. Therefore, the buckling load is equal the sum of the buckling loads of the fuel column and the buckling load of the cladding column. If, however, the bending stiffness of the fuel column suddenly drops to zero, the axial load in the fuel column would be entirely resisted by the bending stiffness of the cladding column. Thus, the total inertia load of the fuel would be resisted by the bending stiffness (buckling resistance) of the cladding column.

Case 3: Axial and lateral displacement compatibility between the fuel column and cladding column is required.

The fuel column is now bonded to the cladding column. (The dowel is glued to the tube.) The inertia load applied to the fuel is now distributed to the fuel and cladding in accordance with the axial stiffness of the fuel column and cladding column. The bending stiffness (buckling resistance) is equal to the EI of the composite section. However, because the columns are concentric, the bending stiffness of the composite section is equal to the sum of the bending stiffnesses of the individual columns, as in Case 2 above. Therefore, the buckling load calculated in Case 2 is the same as the buckling load calculated here in Case 3. 


\section{DERIVATION OF THE CRITICAL BUCKLING LOAD}

The buckling problem to be solved is straight forward, and the solution follows from first principles. The problem involves the buckling of two simply supported concentric columns, i.e., a solid fuel column within a cladding tube column. The fuel column and the cladding column are in lateral contact, but can slide vertically relative to each other. Each column independently supports its own vertical load (weight or "g" load), but both are constrained to deform together so that lateral displacement compatibility is enforced. That is, the fuel column, as it displaces under "g" loading, must remain inside of and in contact with the cladding. The inertia load in the fuel column together with the inertia load in the cladding column and the requirement of displacement compatibility will drive the buckling of the fuel rod.

The basic equation governing the analysis of a beam-column acted upon by an axial compressive force $P$ and a distributed lateral load $q$, as given by Timoshenko [6], is

$$
E I \frac{d^{4} y}{d x^{4}}+P \frac{d^{2} y}{d x^{2}}=q
$$

where $y$ is the lateral displacement of the beam at any location $x$ along the beam, and EI is the flexural rigidity of the beam in the plane of bending. Since the critical buckling load of a column is determined for conditions under which the lateral load vanishes, the differential equation governing the column is

$$
E I \frac{d^{4} y}{d x^{4}}+P \frac{d^{2} y}{d x^{2}}=0
$$

Drop tests and analyses of fuel rods under inertia loading show that buckling takes place between transverse spacer grids in the form of half sine waves with the largest lateral displacements occurring between the fuel assembly head and the first spacer grid closest to the impact end of the rod, as shown in Figure 1. It is assumed in the derivation that the total weight of the fuel and cladding, over the entire length of the rod, is applied to this lower segment of length, $l$.

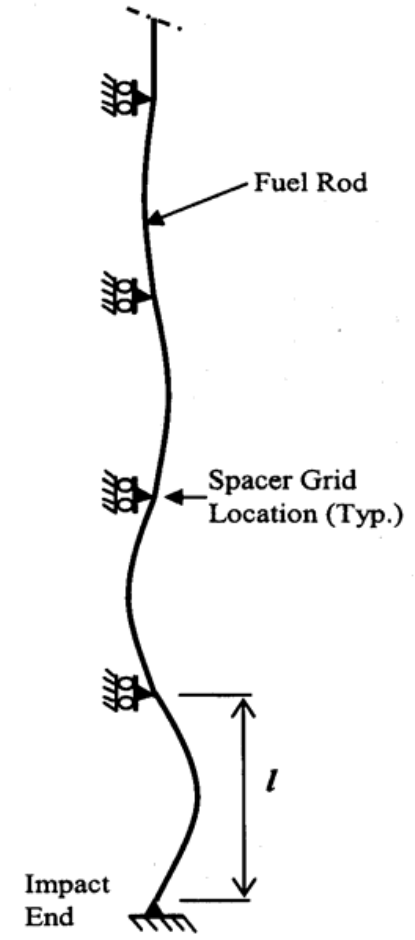

Figure 1: Buckled shape of a fuel rod following drop impact. 
Letting $\alpha$ equal the impact g load magnitude, then the axial inertia load $P_{c}$ in the cladding column and axial inertia load $P_{f}$ in the fuel column are given by

$$
\begin{aligned}
& P_{c}=\alpha W_{c} \\
& P_{f}=\alpha W_{f}
\end{aligned}
$$

where $W_{c}$ and $W_{f}$ are conservatively taken to be equal to the total weight of the cladding and fuel respectively. The fuel and cladding columns are shown separately in Fig. 2. Substituting Eq. (3) into Eq. (2), the governing equation for elastic stability of the cladding column becomes

$$
E_{c} I_{c} \frac{d^{4} y_{c}}{d x^{4}}+\alpha W_{c} \frac{d^{2} y_{c}}{d x^{2}}=0
$$

and for the fuel column is

$$
E_{f} I_{f} \frac{d^{4} y_{f}}{d x^{4}}+\alpha W_{f} \frac{d^{2} y_{f}}{d x^{2}}=0
$$

where the subscripts $c$ and $f$ denote the lateral displacement $y$ and flexural rigidity $E I$ of the cladding and fuel columns respectively.

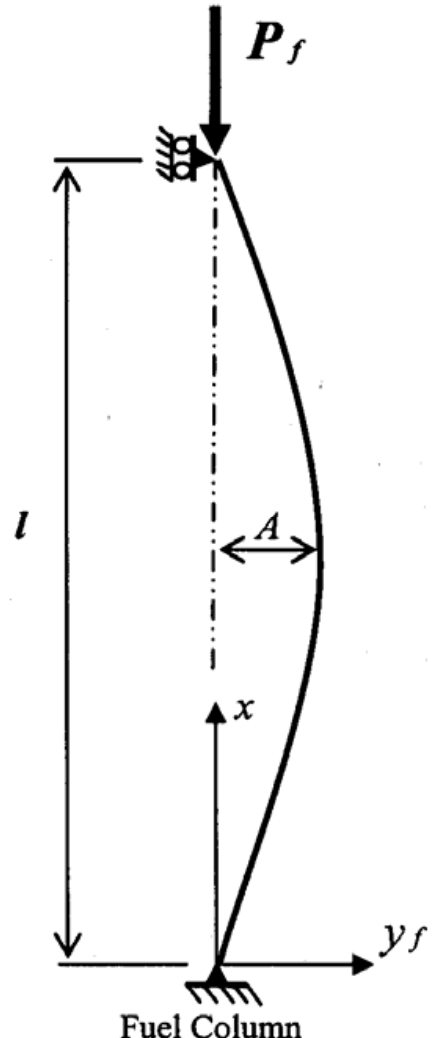

Fuel Column

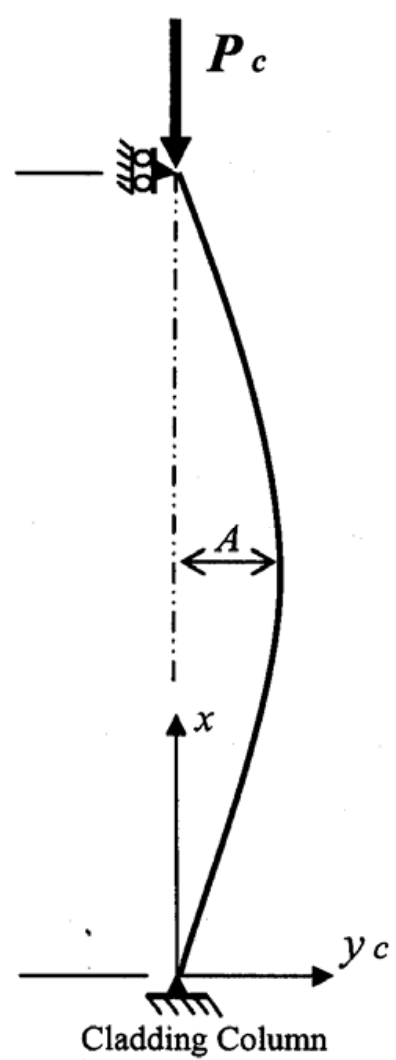

Cladding Column

Figure 2: $\quad$ Fuel pellet column and cladding tube column with compatible lateral displacements, $A$. 
To satisfy displacement compatibility between the cladding and fuel, the lateral displacement at each $\mathrm{x}$ location along the cladding and fuel columns must be equal, and therefore

$$
y_{c}=y_{f}=y
$$

Adding Eq. (4) and Eq. (5) after imposing the displacement compatibility conditions of Eq. (6) one obtains

$$
\left(E_{c} I_{c}+E_{f} I_{f}\right) \frac{d^{4} y}{d x^{4}}+\alpha\left(W_{c}+W_{f}\right) \frac{d^{2} y}{d x^{2}}=0
$$

This is the general differential equation for the buckling of two concentric columns constrained to have the same lateral displacement when each column is subjected to a constant axial force equal to the total inertia load of the column.

Letting

$$
k^{2}=\frac{\alpha\left(W_{c}+W_{f}\right)}{\left(E_{c} I_{c}+E_{f} I_{f}\right)}
$$

Eq. (7) becomes

$$
\frac{d^{4} y}{d x^{4}}+k^{2} \frac{d^{2} y}{d x^{2}}=0
$$

The general solution of Eq. (9) can be written in the form

$$
y=A \sin k x+B \cos k x+C x+D
$$

where constants $A, B, C$ and $D$ are determined from the boundary conditions for the column. For the case of a column with hinged ends the deflection and bending moment are zero at the ends, and hence the boundary conditions are

$$
y=\frac{d^{2} y}{d x^{2}}=0 \quad \text { at } x=0 \text { and } x=l
$$

Applying the four boundary conditions of Eq. (11) to the general solution of Eq. (10) one obtains

$$
\begin{aligned}
& B=C=D=0 \\
& A \sin (k l)=0
\end{aligned}
$$

In order for lateral displacement and buckling to occur $A$ cannot be zero, therefore, $\sin (k l)$ must be zero. The equation

$$
\sin (k l)=0
$$

is satisfied when $k l=n \pi$ which determines the magnitude of the critical load. The smallest critical load occurs when $n=1$, and therefore

$$
k^{2}=\frac{\pi^{2}}{l^{2}}
$$

Substituting Eq. (8) into Eq. (14) gives 


$$
\alpha_{c r}\left(W_{c}+W_{f}\right)=\frac{\pi^{2}}{l^{2}}\left(E_{c} I_{c}+E_{f} I_{f}\right)
$$

Solving for the critical inertia load magnitude $\alpha_{c r}$, Eq. (15) becomes

$$
\alpha_{c r}=\frac{\pi^{2}}{l^{2}} \frac{\left(E_{c} I_{c}+E_{f} I_{f}\right)}{\left(W_{c}+W_{f}\right)}
$$

which determines the critical inertia load magnitude for fuel rod buckling in terms of the flexural rigidity and weight of the fuel and cladding.

Equation (16) defines the critical inertia load magnitude for the buckling of a fuel rod composed of a fuel column that is constrained to remain within the boundary of the cladding tube column. From a practical point of view, however, the flexural rigidity of both new and spent fuel is difficult to determine and subject to large uncertainties. It is therefore reasonable for the purposes of design and evaluation to conservatively neglect the flexural rigidity of the fuel in the calculation of the critical buckling load.

Letting

$$
E_{f} I_{f}=0
$$

in Eq. (16), the final result for the critical inertia load magnitude for the buckling of a fuel rod is

$$
\alpha_{c r}=\frac{\pi^{2}}{l^{2}} \frac{E_{c} I_{c}}{\left(W_{c}+W_{f}\right)}
$$

\section{CONCLUSIONS}

Equations (16) and (18) show that the critical buckling load of a fuel rod under inertia loading depends on both the weight of the cladding and the total weight of the fuel, regardless of whether the fuel is bonded to the cladding or not. Therefore, $100 \%$ of the fuel always participates in the buckling of a fuel rod under inertia loading. Since the fuel $\left(W_{f}\right)$ constitutes about $80 \%$ of the weight of a fuel rod, neglecting the weight of the fuel, or considering only the portion of the fuel that may be bonded to the cladding, can overestimate the critical inertia load magnitude at which buckling initiates by as much as a factor of five.

\section{NOMENCLATURE}

EI Flexural rigidity

$E_{c} I_{c} \quad$ Flexural rigidity of the cladding tube column

$E_{f} I_{f} \quad$ Flexural rigidity of the fuel pellet column

$P \quad$ Axial load in a column

$P_{c} \quad$ Axial load in the cladding column

$P_{f} \quad$ Axial load in the fuel column

$W_{c} \quad$ Total weight of the fuel rod cladding

$W_{f} \quad$ Total weight of the fuel pellets in the fuel rod

$k \quad$ Critical buckling load parameter

$x \quad$ Coordinate along the length of the column

y Displacement perpendicular to the column

$\alpha_{c r} \quad$ Critical inertia load magnitude for rod buckling (g's) 


\section{REFERENCES}

[1] Code of Federal Regulations 10 Part 71, 2003, Office of the Federal Register National Archives and Records Administration, Washington, DC.

[2] Ramsey, C., Monika, W., Schwartz, M., 1987, “Dynamic Impact Effects on Spent Fuel Assemblies,” UCID21246, Lawrence Livermore National Laboratory, Livermore, California.

[3] Sanders, T., Seager, K., Rashid, Y., Barrett, P., Malinauskas, A., Einziger, R., Jordan, H., Duffey, T., Sutherland, S., Reardon, P., 1992, "A Method for Determining the Spent Fuel Contribution to Transport Cask Containment Requirements,” SAND90-2406, Sandia National Laboratories, Albuquerque, New Mexico.

[4] Spent Fuel Project Office, Interim Staff Guidance-12 Revision 1, "Buckling of Irradiated Fuel Under Bottom End Drop Conditions,” 1999, US Nuclear Regulatory Commission, Washington, DC.

[5] Spent Fuel Project Office, “Standard Review Plan for Spent Fuel Dry Storage Facilities,” NUREG-1567, 2000, US Nuclear Regulatory Commission, Washington, DC.

[6] Timoshenko, S., Gere, J., 1961, “Theory of Elastic Stability,” McGraw-Hill, New York, NY. 